\title{
Desempenho dos fundos de Ações Î́ndice Ativos utilizando o CAPM de 1997 a 2019
}

Performance of Active Index Stock funds using the CAPM from 1997 to 2019

\author{
Robert Aldo Iquiapaza ${ }^{1}$ \\ Rodrigo Lage d'Azevedo Carneiro ${ }^{2}$ \\ Hudson Fernandes Amaral ${ }^{3}$ \\ Bruno Pérez Ferreira ${ }^{4}$
}

\begin{abstract}
Resumo
O objetivo foi avaliar o desempenho de fundos de ações índice ativos no mercado brasileiro, ao longo de mais de 21 anos, com base no modelo CAPM, de forma a evidenciar se existem habilidades nos administradores de carteiras desses fundos para superarem o desempenho do índice Ibovespa, controlando a influência do viés de sobrevivência na geração do retorno anormal. $\mathrm{O}$ estudo utilizou dados secundários e abordagem quantitativa. A amostra considerou 588 fundos de ações índice ativos de janeiro 1997 a dezembro 2019. O retorno anormal foi calculado regredindo os retornos mensais dos fundos em relação aos retornos mensais do Ibovespa, ambos em excesso à taxa do CDI. Isto é, utilizando a equação de regressão do modelo CAPM. Os resultados revelaram que os fundos de ações índice ativos analisados, em valores médios e medianos, tiveram desempenho significativo positivo. Do total da amostra $64,6 \%$ dos fundos apresentaram alfa positivo, ou seja, desempenho ajustado ao risco superior ao do Ibovespa no período analisado. Constatou-se que o fator viés de sobrevivência, no longo prazo, exerceu influência considerável nos resultados de desempenho. O desempenho dos fundos descontinuados foi, em média, significativamente inferior ao dos fundos em funcionamento. Esses resultados podem subsidiar outras pesquisas e a tomada de decisões de investidores que desejem compreender a magnitude do retorno anormal dessa classe de fundos em relação ao Ibovespa no longo prazo, e também para auxiliar na seleção desses fundos de ações visando retornos superiores ao proporcionado pelo índice de referência.

Palavras-Chave: Investimentos. Fundos de Investimentos. Fundos de Ações. Gestão Ativa. Gestão de Portfólio.
\end{abstract}

\begin{abstract}
The aim was to evaluate the performance of active index stock funds in the Brazilian market, over more than 21 years, based on the CAPM model, in order to highlight if there exist management skills to outperform the Ibovespa benchmark, controlling for the influence of survivorship bias in the generation
\end{abstract}

\footnotetext{
${ }_{1}^{1}$ Professor Associado, Universidade Federal de Minas Gerais - UFMG, Brasil. ORCID https://orcid.org/0000-0003-1657-2823. E-mail: riquiapaza@gmail.com

${ }_{2}^{2}$ Mestre em Administração/Finanças, Universidade Federal de Minas Gerais - UFMG, Brasil. ORCID https://orcid.org/0000-0002-1956-0564. E-mail: rodrigolagec@gmail.com

${ }^{3}$ Professor Titular aposentado da UFMG, Professor do Centro Universitário Unihorizontes, Brasil. ORCID https://orcid.org/0000-0001-8455-0285. E-mail: hfamaral.cepead@gmail.com ${ }_{4}^{4}$ Professor Associado, Universidade Federal de Minas Gerais - UFMG, Brasil. ORCID https://orcid.org/0000-0002-1011-5253. E-mail: brunoperez.bh@gmail.com
} 
of abnormal returns. The study used secondary data and a quantitative approach. The sample considered 588 active index stock funds from January 1997 to December 2019. The abnormal returns were calculated by regressing the funds' monthly returns against the Ibovespa' monthly returns, both in excess of the CDI rate. That is, using the regression equation of the CAPM model. The results revealed that the analyzed funds, in average and median values, had a positive significant performance. From the total sample, 64.6\% of the funds showed a positive alpha, that is, a risk-adjusted performance superior to that of the Ibovespa in the analyzed period. It was also found that the survival bias factor, in the long run, had considerable influence on the performance results. The performance of the discontinued funds was, on average, significantly lower than that of the funds in operation. These results may support further research and decision making by investors who wish to understand the abnormal returns of these funds relative to the Ibovespa over the long term, and also to assist in the selection of funds aiming at superior returns to those provided by the benchmark index.

Keywords: Investments. Mutual Funds. Equity Funds. Active Management. Portfolio Management.

$$
* * *
$$

\section{Introdução}

Ao contrário da estratégia de gestão passiva, em que o administrador de carteiras investe em ativos, visando reproduzir a carteira de um índice previamente definido, a fim de obter um retorno o mais próximo possível do proporcionado pelo índice, na estratégia de gestão ativa o administrador de carteiras realiza a compra e a venda de ativos com o objetivo de apurar um retorno superior ao do índice fixado como referência para a gestão da carteira ou fundo de investimento (Sharpe, 1991; Castro \& Minardi, 2009). A estratégia de gestão ativa, utilizando diferentes abordagens, conhecimentos e habilidades dos gestores, pode oferecer ao investidor a possibilidade de auferir maiores rendimentos. Porém, faz com que o investidor incorra em uma maior exposição ao risco e aos custos decorrentes de transações frequentes (Sharpe, 1991; Matallín-Sáez, Soler-Domínguez, \& Tortosa-Ausina, 2018).

$\mathrm{Na}$ classe de fundos de ações, tem-se os fundos índice ativos, que são objeto da pesquisa, e que têm como finalidade superar um índice de mercado. Estes fundos "se utilizam de deslocamentos táticos em relação à carteira de referência para atingir seu objetivo" (Anbima, 2015, p.14). Outros fundos de ações ativos como os de carteira livre ou de segmentos e 
indústrias específicos tem um conjunto mais variado de opções de investimento, e procuram agregar valor quando conseguem uma diferenciação e maior exposição ao risco idiossincrático, e isso pode levar a um desempenho anormal diferente de zero (Matallín-Sáez, et al., 2018).

O debate em finanças entre os defensores e os opositores da adoção de estratégias de gestão ativa de fundos de investimento tem sido intenso nas últimas décadas (Jensen, 1968, 1969; Grinblatt \& Titman, 1989; Sharpe, 1991; Malkiel, 1995; Gruber, 1996; Carhart, 1997; Wermers, 2000; Elton, Gruber \& Busse, 2004; Cremers \& Petajisto, 2009; Fama \& French, 2010; Matallín-Sáez, et at., 2018, Casavecchia \& Hulley, 2018; Stark, 2019; Riley, 2021).

A questão tornou-se ainda mais relevante após o lançamento do primeiro fundo de investimento indexado no final da década de 1970 no mercado americano. Observou-se, desde então, a ascensão da gestão passiva e um crescimento da popularidade dos fundos indexados e dos Exchange Traded Funds - ETF's (Cremers, Fulkerson \& Riley, 2019).

A maior parte dos estudos empíricos, realizados acerca do desempenho dos fundos de investimentos no período de 1968 a 1997, aponta para a constatação de que os mercados são eficientes (Fama, 1970; Carhart, 1997), e que, portanto, não existem indícios significativos de que os fundos, que adotam uma estratégia de gestão ativa, consigam obter desempenho superior aos índices de referência do desempenho do mercado ou benchmarks de maneira recorrente.

Um dos primeiros e importantes estudos que constataram que a gestão ativa não cria valor para os investidores foi elaborado por Jensen (1968). Decorridos quase 30 anos, em outro estudo de referência na literatura sobre o assunto, Carhart (1997) chegou a um resultado similar. Os resultados obtidos não evidenciaram a existência de administradores de carteira suficientemente habilidosos ou informados para produzirem desempenho superior de maneira regular. 
Assim, dentre a grande variedade de fundos de ações no mercado, torna-se um desafio ao investidor escolher o melhor fundo para investir e, assim, obter desempenho futuro consistente (Silva, Roma, \& Iquiapaza, 2018). Análises com período de tempo mais longo podem subsidiar na tomada dessa decisão.

A fim de verificar se a chamada sabedoria convencional sobre a gestão ativa continuava válida, Cremers, Fulkerson e Riley (2019) realizaram uma extensa revisão da literatura sobre o tema, desde o trabalho de Carhart (1997). Os autores sugerem que a sabedoria convencional julga a gestão ativa de maneira negativa. Os estudos elaborados nos últimos 20 anos demonstram que os administradores de carteira que adotam estratégias de gestão ativa possuem diversas habilidades e, em muitos casos, conseguem criar valor para os investidores, mesmos após a contabilização das despesas dos fundos (Cremers, Fulkerson \& Riley, 2019). Uma dificuldade, presente em alguns estudos anteriores, é o pequeno tamanho da série temporal utilizado nos testes, o que não permite identificar corretamente a significância estatística do desempenho. Dessa forma, o tamanho da série utilizada na presente pesquisa, permite uma maior confiabilidade sobre o efeito significativo das estratégias utilizadas pelos gestores das carteiras de fundos.

Este artigo analisa o desempenho dos fundos de ações ativos que têm como benchmark o índice de mercado, mensurando o desempenho ajustado ao risco de 588 fundos de ações ativos no mercado brasileiro, e utilizando uma amostra ao longo de mais de 21 anos de informações. Adicionalmente, verificou-se a influência do viés de sobrevivência na geração do retorno anormal ajustado ao risco, e discute as dificuldades enfrentadas pelos administradores de fundos de ações ativos para superarem o desempenho do índice de referência, o Ibovespa.

Assim, são três as principais diferenças e contribuições do artigo: utiliza um período longo de tempo para avaliação de desempenho dos fundos; analisa uma categoria de fundos de ações que individualmente é 
pouco estudada nas pesquisas no Brasil; e, mostra evidências do viés de sobrevivência na estimativa de desempenho desses fundos de forma agregada.

O referencial teórico utilizado para fundamentar a análise realizada está contido na seção 2 deste artigo. A metodologia empregada para o cálculo do desempenho ajustado ao risco dos fundos de ações ativos e os dados selecionados estão contidos na seção 3. Os resultados obtidos são apresentados na seção 4. As considerações finais acerca deste estudo são discutidas na seção 5 deste estudo.

\section{Referencial Teórico}

\subsection{Hipótese da Eficiência dos Mercados}

Segundo a teoria da eficiência dos mercados, um mercado de capitais eficiente é aquele em que os preços correntes dos ativos sempre refletem completamente as informações disponíveis (Fama, 1970). Em um mercado de capitais eficiente, os preços correntes refletem o valor presente subjacente dos ativos. Outra característica fundamental de um mercado de capitais eficiente é que não é possível obter lucros extraordinários utilizando-se as informações disponíveis. A teoria da eficiência dos mercados está particularmente interessada em como a informação afeta os preços dos ativos. A fim de examinar como se dá este ajuste nos preços, são realizados testes empíricos que utilizam três subconjuntos de informações relevantes. Em primeiro lugar, os testes da forma fraca de eficiência consideram os preços históricos. Em seguida, os testes da forma semi-forte de eficiência levam em consideração as informações publicamente disponíveis. Finalmente, os testes da forma forte de eficiência consideram todas as informações disponíveis.

Deseja-se, nos testes da forma fraca, verificar a influência dos preços históricos sobre os preços correntes dos ativos. Um mercado de capitais é eficiente na forma fraca quando os preços dos ativos incorporam completamente os preços históricos. Portanto, o mercado é eficiente em 
relação a estes preços. Qualquer estratégia de investimentos baseada em preços históricos não é capaz de gerar lucros em um mercado eficiente na forma fraca. A compra de ações, utilizando padrões de movimentos de preços passados não é melhor do que a compra de ações de maneira aleatória (Fama, 1970).

Nos testes da forma semi-forte analisa-se o impacto das informações públicas disponíveis sobre os preços dos ativos. Neste caso, um mercado de capitais é eficiente na forma semi-forte quando os preços dos ativos refletem todas as informações públicas, tais como os demonstrativos econômicofinanceiros divulgados pela empresa, o anúncio do desdobramento de ações, dentre outros. Dessa forma, os investidores não conseguem superar o mercado com estratégias de investimento utilizando as informações públicas disponíveis (Fama, 1970).

Nos testes da forma forte o objetivo é investigar o efeito de todas as informações disponíveis, tanto públicas quanto privadas, sobre os preços dos ativos. Um mercado de capitais é eficiente na forma forte quando os preços dos ativos refletem todas as informações existentes. $\mathrm{Na}$ forma forte de eficiência dos mercados, os investidores possuem acesso e utilizam todas as informações que quaisquer indivíduos possuam com relação aos ativos, mesmo em se tratando de informações privilegiadas (insider information) (Fama, 1970; Ross, Westerfield, \& Jaffe, 1995).

Os resultados dos testes empíricos realizados em diferentes mercados evidenciam a validade das hipóteses da eficiência da forma fraca e da eficiência da forma semi-forte. Fama, Fisher, Jensen, e Roll (1969) elaboraram um estudo de eventos que analisou 940 desdobramentos de ações. Retornos anormais positivos foram verificados antes do desdobramento das ações e nos períodos próximos às datas dos anúncios dos desdobramentos, sugerindo assim que os desdobramentos pareciam comunicar informações ao mercado. Após o desdobramento, não foi constatada qualquer tendência de aumento do retorno anormal positivo, o 
que é consistente com a teoria da eficiência dos mercados na forma semiforte.

Fama (1991) afirma que a evidência mais clara sobre a eficiência dos mercados vem dos estudos de eventos, especialmente os estudos de eventos sobre retornos diários. Os resultados apontam que em média os preços das ações se ajustam rapidamente a informações sobre decisões de investimento, mudanças de dividendos, mudanças na estrutura de capital e transações corporativas de controle. Esta evidência possibilita que se chegue à conclusão de que os preços se ajustam eficientemente às informações publicamente disponíveis das firmas.

A hipótese da eficiência da forma semi-forte também pode ser analisada comparando-se o desempenho de fundos de investimento em relação ao desempenho de um índice de referência do mercado. Se o mercado for eficiente na forma semi-forte, então os administradores de carteira, que nos processos de análise e de tomada de decisão de investimentos utilizam, de maneira geral, informações públicas disponíveis, não conseguirão proporcionar retornos médios superiores aos obtidos por um investidor típico (Ross et al., 1995).

Considerando os estudos realizados sobre a forma semi-forte de eficiência, pode-se concluir que a gestão ativa de fundos de investimento, que parte do pressuposto de que existem administradores de carteiras suficientemente talentosos para selecionar ativos sub-avaliados com elevado potencial de valorização, e que se baseia na aplicação de técnicas da análise econômica e/ou estatística, não pode garantir, de forma consistente, a obtenção de desempenho superior ao de um índice de referência do mercado (Carneiro, 2014).

Os resultados dos testes empíricos não conseguiram comprovar a validade da hipótese da forma forte de eficiência. Foram realizados estudos sobre os retornos proporcionados por operações realizadas por insiders das empresas, indivíduos com acesso a informações que não estão disponíveis publicamente. Verificou-se que estas operações foram capazes de gerar 
retornos anormais substanciais, o que atesta que os mercados não são eficientes na forma forte. Apesar dos insiders das empresas possuírem informações privadas que proporcionam a eles retornos anormais em suas operações, os outsiders não podem lucrar com base em informações públicas sobre insider trading (Fama, 1991).

Uma constatação interessante de Fama (1991) é que a literatura sobre mercados eficientes é o primeiro caso em finanças em que a pesquisa acadêmica trouxe uma discussão junto ao mercado financeiro. Antes do trabalho sobre eficiência, acreditava-se que a informação privada era abundante entre os administradores de carteiras. A pesquisa sobre eficiência propôs o desafio que a informação privada é rara. Um resultado do trabalho foi o aumento das estratégias de investimento passivas, em que os administradores de carteiras simplesmente compram e mantem carteiras diversificadas, com o objetivo de reproduzir um índice de referência do mercado. Administradores de carteiras que adotavam estratégias passivas e cobravam, por esta razão, taxas baixas de remuneração, não eram importantes no mercado norte-americano em 1960. Mais recentemente, estes administradores e os fundos passivos se tornaram parte importante da indústria de gestão de investimentos (Gruber, 1996; Castro, \& Minardi, 2009; Cremers, Fulkerson \& Riley, 2019).

A literatura sobre eficiência de mercado também produziu uma demanda por avaliação de desempenho de administradores de carteiras. Segundo Fama (1991), em 1960 os administradores de carteiras eram livres para argumentar sobre os seus desempenhos. Após o trabalho sobre eficiência, a mensuração de desempenho relativamente a benchmarks passivos passou a ser a regra, e existem firmas que se especializaram em avaliar administradores de carteiras. Os dados gerados por estas firmas são, inclusive, uma fonte para testes sobre informações privadas que os acadêmicos têm utilizado com frequência. 


\subsection{Desempenho de Fundos}

Sharpe (1966) analisou o desempenho de 34 fundos de investimento frente ao índice Dow Jones Industrial Average, no período de 1954 e 1963. Foi utilizado como medida de comparação o índice $\mathrm{R} / \mathrm{V}$, que mensura o retorno proporcionado em relação à variabilidade do retorno. Somente 11 fundos de investimento apresentaram desempenho superior ao do portfólio do Dow Jones.

Utilizando os fundamentos do Capital Asset Pricing Model - CAPM, modelo de equilíbrio de um único fator desenvolvido por Sharpe (1964), Lintner (1965) e Mossin (1966), Jensen (1968 e 1969) verificou que os retornos para os investidores em fundos de investimento americanos no período de 1945 a 1965 foram, em média, 1\% ao ano inferiores à linha de mercado, linha que parte da taxa livre de risco até a carteira de mercado representada pelo índice $\mathrm{S} \& \mathrm{P} 500$, e que os retornos médios em mais da metade dos fundos foram abaixo da linha. Somente quando todas as despesas de conhecimento público dos fundos foram adicionadas de volta é que os retornos médios se dispersaram aleatoriamente sobre a linha de mercado. A conclusão de Jensen (1968 e 1969) é que os administradores de carteiras não têm acesso às informações privadas. O administrador de carteiras médio apresentou desempenho inferior ao do índice S\&P 500 agregado, o que é condizente com a teoria da eficiência dos mercados na forma semi-forte.

Estudos realizados por Brinson, Hood, e Beebower (1986) e por outros autores com o objetivo de avaliar o desempenho dos fundos de investimento utilizando modelos multifatoriais constataram que fundos de investimento e fundos de pensão apresentaram retornos anormais negativos frente aos índices de referência utilizados. Novamente, ficou evidenciado que os administradores de carteiras não têm acesso a informações privadas.

Ao analisar os retornos dos fundos de investimento no período de 1971 a 1991, Malkiel (1995) verificou que os fundos apresentaram desempenho inferior ao do mercado, não somente após a dedução das taxas de 
administração, mas também em relação ao resultado bruto de todas as despesas informadas com exceção das taxas de carregamento. Além disso, no contexto do CAPM, não foi constatada qualquer evidência de excesso de retorno e de relacionamento positivo entre risco e retorno.

Gruber (1996) também comprovou a dificuldade dos fundos de investimento em apresentar desempenho superior aos índices de referência de maneira recorrente, utilizando tanto o CAPM, quanto modelos multifatoriais. Os fundos apresentaram, em média, um retorno ajustado ao risco negativo no período de 1985 a 1994.

Utilizando uma amostra sem viés de sobrevivência, Carhart (1997) demonstrou que fatores comuns nos retornos de ações (Fama \& French, 1992) e as diferenças persistentes nas despesas e nos custos de transação explicam quase toda a previsibilidade nos retornos dos fundos de ações. Os resultados encontrados por Carhart (1997) não suportam a existência de gestores suficientemente habilidosos ou informados para produzirem desempenho superior.

Mais recentemente, Fama e French (2015) ampliaram o modelo de 3 fatores, acrescentando os fatores investimento e rentabilidade. Contudo, esse novo modelo, apesar de aumentar o poder explicativo, não oferece o melhor trade-off para avaliação da performance de fundos de investimentos de ações livre brasileiros (Fernandes, Fonseca \& Iquiapaza, 2018).

No Brasil, Borges e Martelanc (2015) construíram uma distribuição empírica para a distribuição dos alfas e compararam com os alfas gerados pelos fundos, na tentativa de diferenciar o desempenho obtido por mera sorte. Na comparação dos alfas simulados com os reais, evidenciaram haver habilidade na geração de retornos anormais positivos, especialmente pelos gestores dos fundos grandes.

De acordo com Silva et al. (2018) e Casavecchia e Hulley (2018) os investidores também deveriam se atentar aos valores das taxas de administração já que estas podem estar negativamente impactando o desempenho e/ou dificultando sua apuração. Outros estudos brasileiros 
corroboram essa ideia (Borges Junior \& Malaquias, 2019; Castro \& Minardi, 2009; Matos, Penna, \& Silva, 2015; Silva, Roma, \& Iquiapaza, 2020).

Outros estudos destacam que os fundos mais jovens, com restrições a resgates, com maior negociação dos ativos da carteira, podem entregar melhor desempenho (Borges Junior \& Malaquias, 2019; Silva et al., 2020). Borges Junior e Malaquias (2019) salientam que os gestores dos fundos mais novos podem ter uma tendência a se esforçar mais em busca de um melhor desempenho, pois estes precisam sobreviver e atrair clientela.

Matallín-Sáez et al. (2018) analisaram a relação entre gestão ativa e performance de uma amostra de fundos de ações ativos americanos, não contaminada pelo viés de sobrevivência, no período de 2001 a 2011, tanto no caso de retornos brutos quanto de líquidos. No agregado, os fundos não obtiveram performance positiva e a maioria deles apresentou performance negativa. Foi possível constatar também que os melhores e os piores fundos apresentaram um nível mais alto de gestão ativa, o que se refletiu na relação em forma de letra "U” entre desempenho e gestão ativa.

Riley (2021) argumenta que os investidores deveriam se preocupar com a performance de um portfólio de fundos ativos e não com a performance de um fundo ativo analisado individualmente. Com base nessa abordagem, Riley (2021) construiu um portfólio de fundos ativos otimizado com baixa volatilidade idiossincrática e obteve um alfa positivo, estatisticamente significante. A performance superior obtida por esse portfólio otimizado não será duradoura, caso o portfólio não seja rebalanceado frequentemente, devido à alocação substancial de capital realizada pelos investidores nos fundos de grande representatividade no portfólio.

Em um estudo realizado com 25 fundos do Paquistão, predominantemente fundos de ações, que comparou o retorno ajustado ao risco dos fundos em relação ao benchmark, Ali, Aqil, Kazmi e Zaman (2021) constataram que os gestores não foram capazes de superar o retorno do mercado. Segundo os autores, fatores qualitativos, como, por exemplo, a 
qualificação dos gestores, instabilidade política e questões macroeconômicas podem ter afetado os retornos dos fundos, de modo que os retornos foram iguais ou inferiores ao retorno do mercado ou de retornos de ativos livres de risco.

\section{Procedimentos Metodológicos}

\subsection{Amostra e Dados}

Este estudo foi elaborado utilizando-se os retornos líquidos mensais, após a cobrança da taxa de administração, de uma amostra de 588 fundos de ações índice ativos, em funcionamento ou já descontinuados, no mercado brasileiro desde janeiro de 1997 a dezembro de 2019, constantes na base de dados SI da Anbima. Os referidos fundos foram selecionados utilizando-se a classificação mais recente "Fundos de Ações Índice Ativos" adotada pela Anbima (2015), instituição que representa as entidades do mercado de capitais brasileiro. Para completar o banco de dados, com um período mais longo de informações, foram consideradas categorias de fundos que já não estão vigentes, mas que corresponderam a fundos ativos referenciados em índices, por exemplo Ibovespa e IBRX ativos, disponíveis na base de dados.

Dessa forma, diferentemente dos estudos anteriores, avaliou-se o desempenho dos fundos de investimento para um longo período, compreendendo 263 meses, mais de 21 anos de informações. Este período proporciona dados suficientes para a elaboração de uma análise adequada. É importante registrar que a amostra de fundos estudada não contém somente fundos em funcionamento, mas também fundos que, porventura, tenham encerrado suas atividades, por quaisquer motivos, no período da análise. Isto se faz necessário para que se evite o chamado viés de sobrevivência. Segundo Elton, Gruber e Blake (1996), o viés de sobrevivência ocorre quando o estudo inclui somente os fundos existentes no final do período da seleção. Ressalta-se ainda que a seleção realizada pode não conter dados de todos os fundos de ações índice ativos em operação ou já descontinuados a partir de 
janeiro de 1997, simplesmente porque alguns fundos não encaminharam suas informações à Anbima, por quaisquer motivos, no período analisado.

A Tabela 1 apresenta informações sobre a representatividade da amostra. No período analisado um total de 1515 fundos estiveram registrados na base de dados, mais de $60 \%$ com menos de 5 anos de informações. Dentro desse total de fundos, 329 constavam no banco de dados como vigentes no período final do estudo. Dessa forma, devido as exigências da técnica de estimação do desempenho de contar ao menos com 60 meses de informações, foram incluídos no estudo somente fundos com mais de 5 anos de informações, resultando na amostra total de 588 fundos, 38,8\% do total de fundos existentes no período. Na amostra definida, um total de 216 fundos estavam vigentes em dezembro de $2019,66 \%$ do total da amostra.

Para a comparação do desempenho dos fundos de ações índice ativos em relação a um índice de referência do mercado, foram levantados na base Economatica ${ }^{\circledR}$ os retornos mensais do Ibovespa, o índice mais importante e tradicional do mercado acionário brasileiro, cuja finalidade básica é a de servir como indicador médio do comportamento do mercado.

A fim de se calcular o prêmio de risco pelo investimento em um ativo arriscado, adotou-se o Certificado de Depósito Interbancário - CDI como referência para o ativo livre de risco, de maneira similar a outros estudos no Brasil (Fonseca, Bressan, Iquiapaza \& Guerra, 2007; Borges \& Martelanc, 2015; Fernandes et al., 2018). A taxa de juros do CDI é o custo do dinheiro que as tesourarias dos bancos utilizam como referência para a realização de operações de captação ou empréstimo de recursos de curto prazo entre os bancos. Os retornos mensais do CDI para o mesmo período de tempo também foram obtidos na base Economatica ${ }^{\circledR}$.

Na Tabela 1 é possível visualizar informações de número de retornos (em anos) para o universo de fundos, os fundos vigentes e os fundos incluídos no estudo, assim como o patrimônio líquido (PL) médio administrado pelos fundos. No universo total percebe-se uma média de PL de $R \$ 71,4$ milhões, já nos fundos vigentes essa média aumenta para $R \$$ 
287,8 milhões, refletindo que os fundos não incluídos no estudo foram principalmente pequenos e de curta duração. $\mathrm{Na}$ indústria de fundos, alguns fundos recentemente lançados pelas administradoras têm um período de incubação com captações iniciais, e não necessariamente permaneceram em funcionamento, ficando vigentes apenas aqueles com retornos atrativos (Borges \& Martelanc, 2015; Malaquias \& Maestri, 2017). Dessa forma, a amostra definida tem fundos com valores de PL bem próximos do universo da classe de fundos analisada.

Tabela 1. População e Amostra dos Fundos de Ações Índice Ativos

\begin{tabular}{|c|c|c|c|c|c|c|c|c|}
\hline \multicolumn{9}{|c|}{ Informações válidas de retornos (anos) } \\
\hline & Mín. & $\begin{array}{c}1^{\circ} \\
\text { Quartil }\end{array}$ & Mediana & Média & $\begin{array}{c}\mathbf{3}^{\mathbf{0}} \\
\text { Quartil }\end{array}$ & Máx. & $\mathbf{N}^{0}{ }_{-}$Fundos & $\begin{array}{c}\text { PL_médio } \\
\text { (Milhões } \\
\text { R\$) }\end{array}$ \\
\hline Total & 0,00 & 1,75 & 3,75 & 5,08 & 7,00 & 21,92 & 1515 & 71,43 \\
\hline Vigentes_Total & 0,00 & 3,33 & 6,92 & 8,31 & 12,17 & 21,92 & 329 & 287,84 \\
\hline Amostra & 5,00 & 6,42 & 8,08 & 9,56 & 11,50 & 21,92 & 588 & 79,75 \\
\hline Vigentes_Amostra & 5,00 & 6,92 & 10,17 & 11,49 & 16,46 & 21,92 & 216 & 292,87 \\
\hline
\end{tabular}

Fonte: Elaborada com dados do estudo.

Notas: $\mathrm{PL}=$ Patrimônio líquido.

\subsection{Cálculo do Desempenho dos Fundos de Ações Ativos}

Grinblatt e Titman (1989) afirmam que a avaliação do desempenho de um investimento é sensível à metodologia empregada. De acordo com Elton, Gruber e Busse (2004), investidores não sofisticados simplesmente verificarão quanto de retorno eles recebem acima ou abaixo do índice de referência como métrica de avaliação dos lucros. Por outro lado, investidores mais sofisticados considerarão o retorno ajustado ao risco como sendo a métrica adequada para a avaliação dos resultados. Nesse sentido, a fim de se verificar se os fundos de ações ativos apresentam desempenho superior ao Ibovespa, será utilizado um modelo de equilíbrio de um único fator, o CAPM, que permite comparar um investimento relativamente a outros, traduzindo a medida de risco em termos de retorno esperado. 
Utilizando-se a equação do CAPM para o cálculo, o retorno anormal de um fundo de ações ativo pode ser quantificado regredindo-se os retornos mensais do fundo em relação aos retornos mensais do índice de referência. $\mathrm{O}$ retorno anormal do fundo, ajustado ao risco, será dado pelo coeficiente linear $\alpha_{\mathrm{p}}$, o ponto em que a linha da regressão linear intercepta o eixo $\mathrm{y}$, também chamado de $\alpha$ de Jensen. A equação de regressão (1) foi utilizada para estimar a medida de desempenho $\left(\alpha_{p}\right)$.

$$
R_{p t}-R_{f t}=\alpha_{p}+\beta_{p}\left(R_{m t}-R_{f t}\right)+\varepsilon_{p t}
$$

em que $R_{p t}$ é o retorno mensal do fundo de ações índice ativo, $R_{f t}$ é o retorno mensal do CDI, $\beta_{\mathrm{p}}$ é a sensibilidade do retorno do fundo de ações ativo em relação ao retorno do Ibovespa, $R_{m t}$ é o retorno mensal do Ibovespa e $\varepsilon_{p t}$ é o termo de erro do modelo.

Os retornos foram calculados usando a diferença do logaritmo do valor das cotas ao final de cada mês, conforme a equação (2).

$$
R_{t}=\ln \left(\frac{v_{t}}{v_{t-1}}\right)
$$

O alfa de Jensen (Jensen, 1968, 1969) foi utilizado para calcular o retorno anormal de um fundo de investimento ajustado ao risco. Esse indicador possibilita verificar se o administrador de carteira apresenta performance superior ao desempenho do mercado, informando, ainda, a magnitude desta performance, sendo a mesma superior ou inferior.

Trata-se de uma medida de seletividade, ou seja, que procura medir a habilidade do administrador de carteira em escolher adequadamente ativos individuais, antecipando períodos de altas nos preços desses ativos (Stark, 2019). Nesse sentido, o alfa de Jensen estima quanto a habilidade de previsão de um administrador de carteira contribui para os retornos de um fundo (Jensen, 1968). 
A opção pelo modelo de um único fator foi fundamentada no tipo de fundos incluídos na amostra, todos fundos ativos referenciados no índice, sendo assim consistentes com o benchmark dos fundos (Ali et al., 2021). Além disso, Fernandes et al. (2018) ao analisar o desempenho dos modelos de precificação em fundos de ações livres reportam que aproximadamente $56 \%$ da variabilidade dos retornos desses fundos podem ser explicados pelo modelo CAPM, e ao incluir outros fatores, como os de Carhart (1997) e Fama e French (2015), o ganho em poder explicativo foi de $2 \%$ a $3 \%$.

\section{Análise dos Resultados}

\subsection{Desempenho dos Fundos de Ações Índice Ativos}

O objetivo de um fundo de ações ativo é que seu retorno anormal ajustado ao risco, medido pelo $\alpha$, seja positivo de forma recorrente. A Tabela 2 apresenta um resumo das estatísticas relacionadas ao desempenho dos 588 fundos de ações índice ativos incluídos neste estudo. No painel da esquerda apresentam-se as estatísticas descritivas dos retornos dos fundos e os indicadores de referência, índice Ibovespa (Ibov) e taxa do CDI. Observase que a média e mediana foram positivas $(0,76 \%$ e $0,89 \%$ mensal, respectivamente) e inferiores ao retorno do mercado e da taxa livre de risco.

No painel da direita (Tab. 2) apresenta-se os indicadores da regressão usando o modelo CAPM. Dessa forma, o desempenho ajustado ao risco, o $\alpha$ atingiu, em média $0,02 \%$, mediana de $0,12 \%$ e variou de $-3,91 \%$ a $1,45 \%$. Estes resultados evidenciam que os fundos de ações índice ativos operando no mercado brasileiro têm, em valores médios, cumprido com seus objetivos, indo de encontro com os resultados dos estudos citados anteriormente (Jensen, 1969; Carhart, 1997), mas alinhados com estudos mais recentes como Borges e Martelanc (2015) e Silva et al. (2020). Embora a gestão ativa em fundos seja uma espécie de jogo de soma zero, existem diferentes situações, como alteração do indexador, mudanças na competição da indústria, alterações de taxas de juros, etc., onde os gestores ativos podem antecipar bons resultados, principalmente com habilidades de seleção de 
ativos e/ou market timing (Hoberg, Kumar, \& Prabhala, 2018; Cremers et al., 2019). Dessa forma, pesquisas recentes descobriram que "muitos gestores ativos têm habilidades observáveis significativas, que essas habilidades criam valor real para os investidores e que essas habilidades persistem com o tempo" (Cremers et al., 2019, p. 11).

O R2 médio foi de 81\%, (variando entre 0,14\% e 99,14\%) indicando que o modelo de equilíbrio empregado neste estudo, o CAPM, apresentou um poder explanatório adequado para a maior parte de fundos.

Tabela 2. Resumo das estatísticas dos retornos dos fundos e do modelo de regressão

\begin{tabular}{lcccccccc}
\hline & \multicolumn{2}{c}{ Retornos mensais (\%) } & \multicolumn{5}{c}{ Indicadores da Regressão } \\
\hline Estatística & Fundos & Ibov & CDI & $\begin{array}{c}\text { Alfa } \\
(\%)\end{array}$ & Beta & $\begin{array}{c}\text { Erro-Pad } \\
\text { Reg(\%) }\end{array}$ & R2 (\%) & $\begin{array}{c}\text { N } \\
\text { Observações }\end{array}$ \\
\hline Média & 0,76 & 1,10 & 1,12 & 0,02 & 0,84 & 2,58 & 81,07 & 114,70 \\
\hline Desvio-padrão & 6,83 & 8,29 & 0,50 & 0,57 & 0,18 & 2,30 & 18,71 & 50,15 \\
\hline Mínimo & $-91,62$ & $-50,34$ & 0,37 & $-3,91$ & 0,03 & 0,53 & 0,14 & 60,00 \\
\hline $1^{\text {o quartil }}$ & $-2,80$ & $-3,32$ & 0,79 & $-0,12$ & 0,74 & 1,56 & 75,60 & 77,00 \\
\hline Mediana & 0,89 & 1,21 & 1,03 & 0,12 & 0,87 & 2,06 & 87,61 & 97,00 \\
\hline $3^{\text {o quartil }}$ & 4,88 & 6,54 & 1,40 & 0,28 & 0,94 & 2,80 & 93,48 & 138,00 \\
\hline Máximo & 52,41 & 21,55 & 3,24 & 1,45 & 1,88 & 25,14 & 99,14 & 262,00 \\
\hline Assimetria & $-1,23$ & $-1,30$ & 1,26 & $-2,91$ & 0,21 & 5,60 & $-2,01$ & 1,19 \\
\hline Curtose & 10,64 & 5,84 & 2,26 & 13,72 & 4,87 & 41,39 & 4,33 & 0,60 \\
\hline No Obs & 67441 & 262 & 262 & 588 & 588 & 588 & 588 & 588 \\
\hline
\end{tabular}

Fonte: Análises realizadas pelos autores.

Notas: R2 = grau de ajuste ou coeficiente de determinação, Erro-Pad Reg = erro padrão da regressão.

Com relação a distribuição da medida de desempenho, a Figura 1 apresenta o histograma com a distribuição do alfa de Jensen, destacando os estatisticamente diferentes de zero ao nível de 5\%, positivos (cor verde) e negativos (cor vermelha). Somente $4,4 \%$ dos fundos tiveram um alfa menor a $-1,0 \%$, mas em $64,6 \%$ dos fundos o desempenho foi positivo. No outro extremo, somente $1 \%$ dos fundos tiveram alfa maior a $1,0 \%$, revelando a dificuldade em se obter desempenhos extraordinários. Do total de fundos, 
$17,2 \%$ apresentaram alfa positivo e significativo ao nível de 5\%, e 9,2\% dos fundos apresentaram alfa negativo e significativo estatisticamente.

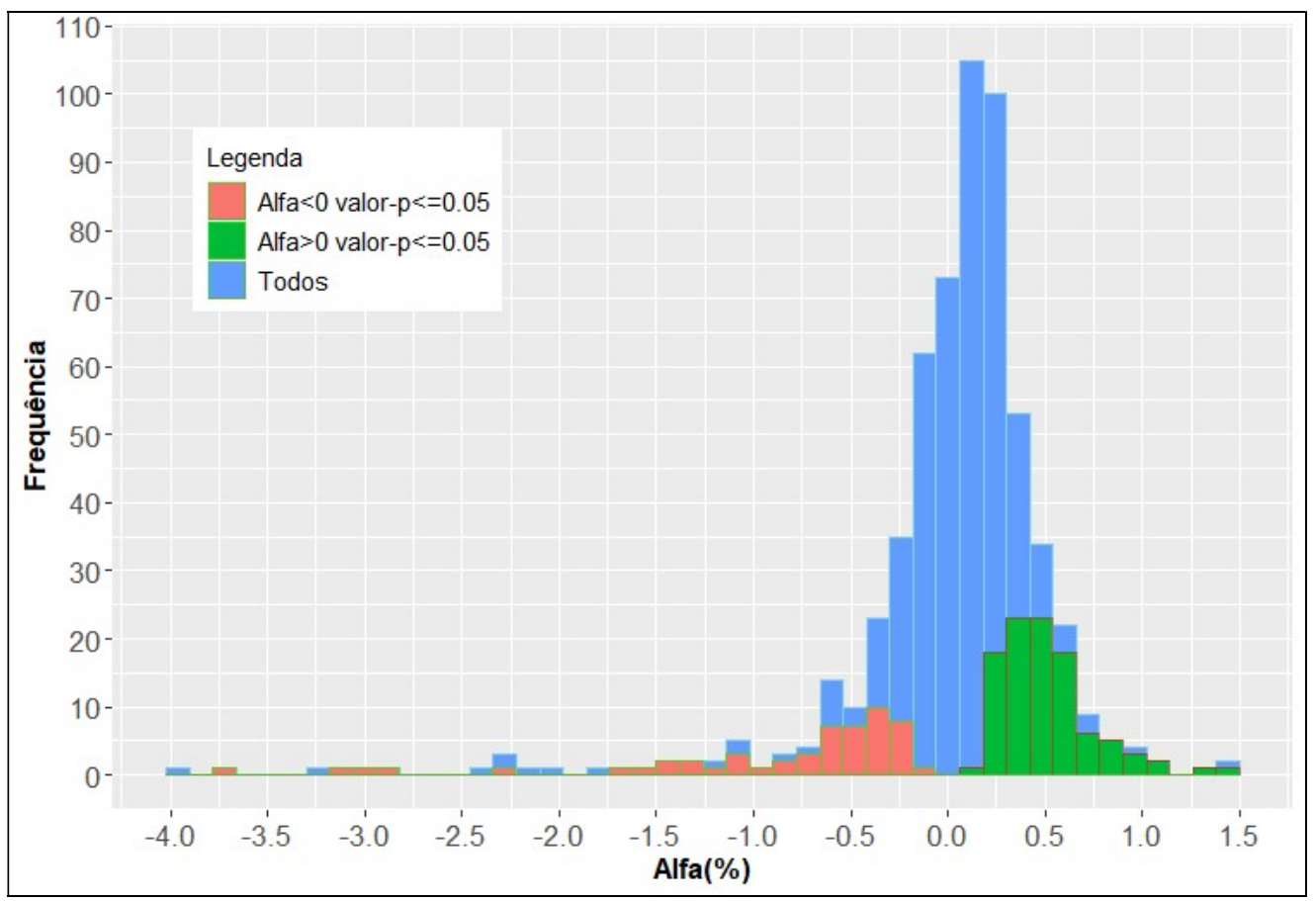

Figura 1. Histograma dos alfas estimados pelo modelo CAPM

4.2 Viés de Sobrevivência no Desempenho dos Fundos de Ações Índice Ativos

A fim de verificar a influência do viés de sobrevivência no desempenho ajustado ao risco dos fundos de ações ativos selecionados neste estudo, os fundos foram segregados em dois grupos, conforme segue: um grupo contendo somente os 216 fundos em funcionamento em dezembro de 2019 e outro grupo contendo os 372 fundos descontinuados, por razões diversas, durante o período do estudo. Observa-se na Tabela 3 que o desempenho ajustado ao risco do grupo composto por todos os fundos "vigentes" foi, em média de $0,12 \%$, e mediana de $0,15 \%$, superior ao desempenho dos fundos em "descontinuados", que, em média ficou em 0,04\%, e mediana de 0,08\%. Utilizando o teste $t$, e o teste não paramétrico de Wilcoxon, em ambos os casos os valores médios foram estatisticamente 
diferentes de zero ao nível de 1\%, assim como os retornos das duas amostras foram estatisticamente diferentes ao mesmo nível de significância. Isso demonstra que a exclusão dos fundos descontinuados pode melhorar significativamente o desempenho apresentado pelo grupo composto pelos fundos em funcionamento, e revela a importância de se manter os fundos descontinuados em análises de desempenho de fundos de investimento (Carhart, 1997; Elton, Gruber, \& Blake, 1996). Por outro lado, entre os fundos descontinuados, observa-se uma maior dispersão dos diferentes indicadores, como o alfa, beta e R2. Assim, com base nestes resultados, o fator viés de sobrevivência exerceu influência considerável no desempenho dos fundos analisados neste estudo.

Tabela 3. Influência do Viés de Sobrevivência no desempenho dos fundos

\begin{tabular}{|c|c|c|c|c|c|c|c|c|}
\hline Situação & Indicador & Média & $\begin{array}{l}\text { Desvio- } \\
\text { padrão }\end{array}$ & Mínimo & Mediana & Máximo & Assimetria & Curtose \\
\hline \multirow{4}{*}{$\begin{array}{l}\text { Vigentes } \\
\text { em dez } \\
2019(216 \\
\text { fundos) }\end{array}$} & Alfa $(\%)$ & 0,12 & 0,41 & $-2,86$ & 0,15 & 1,45 & $-2,54$ & 17,55 \\
\hline & Beta & 0,83 & 0,15 & 0,42 & 0,87 & 1,42 & $-0,40$ & 0,67 \\
\hline & R2 (\%) & 84,03 & 15,74 & 4,65 & 90,07 & 99,14 & $-2,08$ & 5,68 \\
\hline & N Obs. & 137,77 & 62,30 & 60,00 & 122,00 & 262,00 & 0,57 & $-1,04$ \\
\hline \multirow{4}{*}{$\begin{array}{c}\text { Descontin } \\
\text { uados (372 } \\
\text { fundos) }\end{array}$} & Alfa(\%) & $-0,04$ & 0,64 & $-3,91$ & 0,08 & 1,27 & $-2,74$ & 10,99 \\
\hline & Beta & 0,84 & 0,20 & 0,03 & 0,88 & 1,88 & 0,31 & 5,04 \\
\hline & R2 (\%) & 79,35 & 20,05 & 0,14 & 86,90 & 98,50 & $-1,90$ & 3,47 \\
\hline & N Obs. & 101,30 & 35,22 & 60,00 & 90,00 & 228,00 & 1,07 & 0,44 \\
\hline
\end{tabular}

Fonte: Análises realizadas pelos autores.

Notas: R2 = grau de ajuste ou coeficiente de determinação, N Obs = número de observações.

Do total de 216 fundos em funcionamento, 1,4\% apresentaram um desempenho ajustado ao risco inferior a -1\%, 72,7\% tiveram alfa positivo, e $1,9 \%$ dos fundos apresentaram alfa superior a $1 \%$ ao mês. Entre esses fundos, $26,4 \%$ tiveram alfa positivo e significativo ao nível de $5 \%$, e $9,7 \%$ dos fundos tiveram alfa negativo e significativo. O R2 médio dos fundos em funcionamento foi de $84,0 \%$.

Do total de 372 fundos descontinuados, 6,2\% apresentaram um desempenho ajustado ao risco inferior a $-1 \%, 60,0 \%$ tiveram alfa positivo, e $0,5 \%$ dos fundos apresentaram alfa superior a $1 \%$ ao mês. Desses fundos, 
$11,8 \%$ tiveram alfa positivo e significativo ao nível de 5\%, e somente em 8,9\% tiveram alfa negativo e significativo. O R2 médio desses fundos foi de $79,2 \%$. Comparando com os fundos em funcionamento percebe-se resultados piores para o desempenho dos fundos descontinuados.

$\mathrm{Na}$ Tabela 4 apresenta-se os resultados para os 10 piores e 10 melhores desempenhos dos fundos vigentes em dezembro de 2019, no caso do pior fundo o resultado foi significativo a $5 \%$ e aparentemente esse desempenho está refletivo na escolha de se afastar do Ibovespa, já que apresentou um beta de 0,48 e R2 de 4,7\%. Já para o melhor fundo o desempenho somente foi significativo ao nível de 10\%, e o resultado também parece ter sido originado em estratégias de menor exposição ao mercado, já que o fundo teve beta de 0,45 e R2 de 9,8\%. Em ambos os casos, pelo valor relativamente baixo do beta, pode-se inferir que, os gestores desses fundos utilizaram estratégias que conduziram a uma composição da carteira diferente, ou em proporções bem distintas, do índice Ibovespa.

Tabela 4. Desempenho dos Fundos de Ações Índice Ativos em Funcionamento

\begin{tabular}{lcccccc}
\hline \multicolumn{1}{c}{ Fundo } & Alfa & Pval-Alfa & Beta & Pval-Beta & R2 & N \\
\hline Figi Fundo De Investimento De Acoes & $-2,8601$ & 0,0245 & 0,4788 & 0,0295 & 4,65 & 102 \\
\hline Fact Active FI Em Acoes & $-2,2982$ & 0,1080 & 1,0818 & 0,0000 & 24,33 & 63 \\
\hline Alfa Fic De FI Em Acoes & $-1,2442$ & 0,0000 & 0,9666 & 0,0000 & 93,97 & 153 \\
\hline Alfamais FIC De FI Em Acoes & $-0,7740$ & 0,0000 & 0,9617 & 0,0000 & 95,68 & 262 \\
\hline Maitaca Acoes FIC FIA & $-0,6172$ & 0,1520 & 0,7956 & 0,0000 & 65,51 & 67 \\
\hline Bradesco FIC De Fia Iv & $-0,6064$ & 0,0000 & 0,9273 & 0,0000 & 95,68 & 262 \\
\hline Santander FC FI Onix Acoes & $-0,5089$ & 0,0000 & 0,9537 & 0,0000 & 97,75 & 159 \\
\hline Bradesco FIC De Fia Maxi & $-0,4550$ & 0,0000 & 0,9109 & 0,0000 & 97,20 & 247 \\
\hline Bradesco FIC De Fia & $-0,4134$ & 0,0002 & 0,8675 & 0,0000 & 94,30 & 262 \\
\hline Banrisul Performance FI Acoes & $-0,3993$ & 0,0770 & 1,0521 & 0,0000 & 82,12 & 218 \\
\hline Uv Equity Brasil FI De Acoes & 0,5812 & 0,0009 & 0,5810 & 0,0000 & 77,50 & 136 \\
\hline Xp Investor FI De Acoes & 0,6033 & 0,0056 & 0,9163 & 0,0000 & 81,92 & 160 \\
\hline M. Safra Equities FI De Acoes & 0,6403 & 0,0428 & 0,5486 & 0,0000 & 43,81 & 126 \\
\hline Reach FI Acoes & 0,7522 & 0,0326 & 0,4831 & 0,0000 & 53,49 & 61 \\
\hline Atmos Institucional FC FI De Acoes & 0,7557 & 0,0052 & 0,6457 & 0,0000 & 68,65 & 89 \\
\hline Atlas One Master FI De Fia & 0,8091 & 0,0010 & 0,5211 & 0,0000 & 55,47 & 120 \\
\hline Alaska Black FIC FI Acoes Bdr Nivel I & 1,0050 & 0,0517 & 1,0800 & 0,0000 & 57,60 & 108 \\
\hline Atmos Institucional Master Fia & 1,0551 & 0,0025 & 0,7818 & 0,0000 & 72,96 & 61 \\
\hline Alaska Black Master FI Acoes Bdr Nivel I & 1,4097 & 0,0110 & 1,1437 & 0,0000 & 55,86 & 112 \\
\hline Dvg1 FI Em Acoes & 1,4539 & 0,0692 & 0,4452 & 0,0018 & 9,75 & 97 \\
\hline Fonte: Análises realizadas pelos autores. & & & & & & \\
Notas: Pval-Alfa = valor-p do alfa, Pval-Beta = valor-p do beta, R2= grau de ajuste ou coeficiente de determinação, \\
N = número de observações. & & & & & &
\end{tabular}


$\mathrm{Na}$ Tabela 5 pode-se verificar os resultados para os 10 piores e 10 melhores desempenhos dos fundos descontinuados ao longo do período, cujos retornos tenham se iniciado em janeiro de 1997. No caso do pior fundo o resultado foi significativo a 10\% e aparentemente esse desempenho está refletivo na escolha de alavancar a exposição ao Ibovespa, já que apresentou um beta de 1,58 e R2 de 22,9\%. Já para o melhor fundo o desempenho somente foi significativo ao nível de $5 \%$, e o resultado foi determinado pela menor exposição ao Ibovespa, já que o fundo teve beta de 0,47 e R2 de $50,0 \%$. Observando-se os alfas dos piores fundos, pode-se inferir que alguns deles podem ter sido descontinuados por desempenho não satisfatório.

Tabela 5. Desempenho dos Fundos de Ações Índice Ativos Descontinuados

\begin{tabular}{lcccccc}
\hline Fundo & Alfa & $\begin{array}{c}\text { Pval- } \\
\text { Alfa }\end{array}$ & Beta & $\begin{array}{c}\text { Pval- } \\
\text { Beta }\end{array}$ & R2 & $\begin{array}{c}\text { No } \\
\text { Obs }\end{array}$ \\
\hline Gwi Fundo De Investimento De Acoes & $-3,9058$ & 0,0536 & 1,5828 & 0,0000 & 22,90 & 70 \\
\hline Gwi Leverage FI Em Acoes & $-3,6739$ & 0,0352 & 1,7655 & 0,0000 & 19,74 & 170 \\
\hline Gwi High Value FI Em Acoes & $-3,2760$ & 0,2036 & 1,0259 & 0,0276 & 4,90 & 99 \\
\hline Elite Vip FIA & $-3,1058$ & 0,0153 & 1,6211 & 0,0000 & 45,24 & 66 \\
\hline Geracao FIA Gol Sul & $-2,9653$ & 0,0000 & 1,2956 & 0,0000 & 88,92 & 66 \\
\hline Treviso FIA Acoes Ie & $-2,3793$ & 0,0501 & 0,7302 & 0,0006 & 14,51 & 78 \\
\hline FIA Gwi Private Investimento No Exterior & $-2,3286$ & 0,1351 & 1,6334 & 0,0000 & 36,85 & 89 \\
\hline Gf FIA Gol Vida & $-2,2702$ & 0,0000 & 1,2377 & 0,0000 & 88,99 & 66 \\
\hline Roma Acoes FI De Acoes & $-2,1903$ & 0,0954 & 0,1843 & 0,4199 & 1,00 & 67 \\
\hline Gwi Small E Mid Caps FI De Acoes & $-1,9816$ & 0,4248 & 0,5281 & 0,2353 & 1,71 & 84 \\
\hline Xp Absoluto Consumo FI De Acoes & 0,7419 & 0,0529 & 0,6905 & 0,0000 & 61,38 & 100 \\
\hline Alaska Poland FI Acoes BDR Nivel I Ie & 0,7823 & 0,0320 & 0,6158 & 0,0000 & 38,54 & 185 \\
\hline Everest Fundo De Investimento De Acoes & 0,8065 & 0,0321 & 0,8194 & 0,0000 & 77,40 & 67 \\
\hline Explora Long Acoes FI Acoes & 0,8092 & 0,0392 & 0,9580 & 0,0000 & 75,97 & 93 \\
\hline Tuias Fi Em Cotas De FI De Acoes & 0,8999 & 0,0399 & 0,6989 & 0,0000 & 64,87 & 71 \\
\hline Fundo De Investimento Elo Acoes Ie & 0,9058 & 0,0022 & 0,6670 & 0,0000 & 65,42 & 124 \\
\hline Cshg Leopardo Acoes FI Acoes & 0,9591 & 0,0234 & 0,7009 & 0,0000 & 70,41 & 63 \\
\hline Bogari Value FC FI De Acoes & 0,9983 & 0,0004 & 0,5783 & 0,0000 & 67,81 & 101 \\
\hline Schroder Brasil FIA & 1,1113 & 0,0135 & 0,7183 & 0,0000 & 79,09 & 64 \\
\hline FI Acoes Lumina & 1,2748 & 0,0016 & 0,4666 & 0,0000 & 49,69 & 75 \\
\hline Font: Anaises realizadas pelos autores. & & & & & &
\end{tabular}

Fonte: Análises realizadas pelos autores.

Notas: Pval-Alfa $=$ valor-p do alfa, Pval-Beta $=$ valor-p do beta, $\mathrm{R} 2=$ grau de ajuste ou coeficiente de determinação, $\mathrm{N}=$ número de observações.

\section{Considerações Finais}

$\mathrm{O}$ artigo teve como objetivo avaliar o desempenho de fundos de ações índice ativos no mercado brasileiro, com base no modelo CAPM, e apresenta evidências de retorno anormal, indicando que existem habilidades nos 
administradores de carteiras desses fundos para superarem o desempenho do índice Ibovespa.

Este estudo contribui com o avanço da literatura da área de três formas. Em primeiro lugar, utilizou-se um período longo de tempo para avaliação de desempenho dos fundos; em segundo lugar, estuda uma categoria de fundos de ações que individualmente é pouco estudada nas pesquisas no Brasil; e finalmente, mostra evidências do viés de sobrevivência na estimativa de desempenho desses fundos de forma agregada.

O período da análise iniciou-se em janeiro de 1997 e terminou em dezembro 2019, compreendendo assim 21 anos e 11 meses ou 263 meses de retornos. Um período de tempo maior ao utilizado por grande parte de estudos recentes nessa área, conferindo maior robustez aos resultados e inferências. O retorno anormal foi estimado com base em um modelo de equilíbrio de um único fator, o CAPM, para a classe de fundos de ações índice ativos no mercado brasileiro. A análise foi realizada em uma amostra de 588 fundos, com um mínimo de 60 meses de informações, em funcionamento ou já descontinuados. A influência do viés de sobrevivência na estimação do desempenho médio dos fundos foi evidenciada comparando fundos vigentes com fundos descontinuados, e o resultado evidenciou as dificuldades enfrentadas pelos administradores de carteiras de fundos de ações ativos para superarem, de forma recorrente, o desempenho do índice de referência, o Ibovespa.

Verificou-se que os fundos de ações índice ativos conseguiram, no período analisado, desempenho médio positivo significativo mensurado pelo alfa de Jensen (média de $0,02 \%$ e mediana de $0,12 \%$ mensal), mostrando que alguns fundos conseguiram retornos superiores ao referencial de mercado. Mas a distribuição do desempenho foi assimétrica, com alguns fundos com perdas significativas no extremo inferior da distribuição, sinalizando a dificuldade para superar, de forma recorrente, o desempenho do Ibovespa. Embora, de um total de 588 fundos constantes na análise, 64,6\% dos fundos 
conseguiram apresentar retorno anormal positivo ajustado ao risco no período analisado. Esse resultado é importante e mostra que alguns gestores de fundos podem estar apresentando habilidade na seleção de carteiras de ativos, conforme destacado em estudos anteriores (Borges \& Martelanc, 2015 e Silva et al., 2020).

Constatou-se também que a exclusão dos fundos descontinuados da análise pode melhorar significativamente o desempenho apresentado pelo grupo de fundos em funcionamento, levando a conclusões incorretas sobre o desempenho. Com base nestes resultados, foi verificado que o fator viés de sobrevivência exerceu influência considerável no período deste estudo. Como relatado pela literatura, os fundos descontinuados apresentaram, em média, um desempenho significativamente inferior ao dos fundos em funcionamento, o que sugere que alguns fundos descontinuados podem ter sido encerrados devido a problemas de desempenho (Elton, Gruber, \& Blake, 1996).

Os resultados do estudo podem subsidiar outros estudos sobre o desempenho dos fundos de investimento, e a tomada de decisões de investidores que desejem compreender a magnitude do retorno anormal dos fundos de ações índice ativos em relação ao Ibovespa. As considerações contidas neste artigo também podem ser úteis para investidores mais sofisticados, que avaliam o desempenho de seus investimentos com base no retorno ajustado ao risco como métrica para medir seus resultados, e definições alternativas de investimento como as destacadas por Riley (2021).

Como aprofundamentos do estudo, podem ser destacados a utilização de modelos de fatores para a mensuração do desempenho (Fama, 1991; Carhart, 1997). Nesse sentido, para que se possam obter resultados ainda mais representativos, novos estudos serão necessários para avaliar o desempenho dos fundos de ações índice ativos utilizando modelos fatoriais. Pode-se também estimar o desempenho por subperíodos, e/ou associá-los a características dos fundos de forma a determinar características internas e 
externas relacionadas aos fundos, que possam favorecer ou prejudicar o seu desempenho (Silva et al., 2020).

\section{Referências}

Ali, M. A., Aqil, M., Kazmi, S. H. A., \& Zaman, S. I. (2021). Evaluation of risk adjusted performance of mutual funds in an emerging market.

International Journal of Finance \& Economics. 1-16.

https://doi.org/10.1002/ijfe.2486

Anbima, Associação Brasileira das Entidades dos Mercados Financeiro e de Capitais, (2015). Classificação de Fundos: Visão geral e nova estrutura. 19p.

Borges, E. C., \& Martelanc, R. (2015). Sorte ou habilidade: uma avaliação dos fundos de investimento no Brasil. Revista de Administração, 50(2), 196207. https://doi.org/10.5700/rausp1194

Borges Junior, D. M., \& Malaquias, R. F. (2019). Restrições de resgate em fundos de ações, liquidez dos ativos e desempenho. Revista de Administração de Empresas, 59(1). https://doi.org/10.1590/S0034-759020190105

Brinson, G. P., Hood, L. R., \& Beebower, G. L. (1986). Determinants of portfolio performance. Financial Analysts Journal, 42(4), 39-44.

Castro, B. R., \& Minardi, A. M. A. F. (2009). Comparação do Desempenho dos Fundos de Ações Ativos e Passivos. Revista Brasileira de Finanças, 7(2), $1-13$.

Carhart, M. M. (1997). On persistence in mutual fund performance. The Journal of Finance, 52(1), 57-82. https://doi.org/10.2307/2329556

Carneiro, R. (2014). A influência da certificação dos administradores de carteira brasileiros no desempenho de fundos de investimento. Dissertação de Mestrado em Administração. Universidade Federal de Minas Gerais.

Casavecchia, L., \& Hulley, H. (2018). Are mutual fund investors paying for noise? International Review of Financial Analysis, 58, 8-23.

https://doi.org/10.1016/j.irfa.2018.04.002

Cremers, K. M., \& Petajisto, A. (2009). How active is your fund manager? A new measure that predicts performance. The Review of Financial Studies, 22(9), 3329-3365. https://doi.org/10.1093/rfs/hhp057

Cremers, K. M., Fulkerson, J. A., \& Riley, T. B. (2019). Challenging the conventional wisdom on active management: A review of the past 20 years 
of academic literature on actively managed mutual funds. Financial Analysts Journal, 75(4), 8-35.

https://doi.org/10.1080/0015198X.2019.1628555

Elton, E. J., Gruber, M. J., \& Blake, C. R. (1996). Survivor bias and mutual fund performance. The Review of Financial Studies, 9(4), 1097-1120.

Elton, E. J., Gruber, M. J., \& Busse, J. A. (2004). Are Investors Rational? Choices among Index Funds. The Journal of Finance, 59(1), 261-288. https://doi.org/10.1111/j.1540-6261.2004.00633.x

Fama, E. F. (1970). Efficient Capital Markets: A Review of Theory and Empirical Work. Journal of Finance, 25(2), 383-417.

https://doi.org/10.2307/2325486

Fama, E. F. (1991). Efficient capital markets: II. The Journal of Finance, 46(5), 1575-1617. https://doi.org/10.2307/2328565

Fama, E. F., \& French, K. R. (2010). Luck versus skill in the cross-section of mutual fund returns. The Journal of Finance, 65(5), 1915-1947.

https://doi.org/10.1111/j.1540-6261.2010.01598.x

Fama, E. F., \& French, K. R. (1992). The Cross-Section of Expected Stock Returns. The Journal of Finance, 47(2), 427-465.

https://doi.org/10.2307/2329112

Fama, E. F., \& French, K. R. (2015). A five-factor asset pricing model. Journal of Financial Economics, 116(1), 1-22.

https://doi.org/10.1016/j.jfineco.2014.10.010

Fama, E. F., Fisher, L., Jensen, M. C., \& Roll, R. (1969). The adjustment of stock prices to new information. International Economic Review, 10(1), 1-21. https://doi.org/10.2307/2525569

Fonseca, N. F., Bressan, A. A., Iquiapaza, R. A., \& Guerra, J. P. (2007). Análise do Desempenho Recente de Fundos de Investimento no Brasil. Contabilidade Vista \& Revista, 18(1), 95-116.

Fernandes, A. R. D. J., Fonseca, S. E., \& Iquiapaza, R. A. (2018). Modelos de mensuração de desempenho e sua influência na captação líquida de fundos de investimento. Revista Contabilidade \& Finanças, 29(78), 435-451. https://doi.org/10.1590/1808-057x201805330

Grinblatt, M., \& Titman, S. (1989). Mutual fund performance: An analysis of quarterly portfolio holdings. Journal of Business, 62(3), 393-416. 
Gruber, M. J. (1996). Another Puzzle: The Growth in Actively Managed Mutual Funds. The Journal of Finance, 51(3), 783-810. https://doi.org/10.1111/j.1540-6261.1996.tb02707.x

Hoberg, G., Kumar,N., \& Prabhala, N. (2018). Mutual fund competition, managerial skill, and alpha persistence. The Review of Financial Studies, 31(5), 1896-1929. https://doi.org/10.1093/rfs/hhx127

Jensen, M. C. (1968). The performance of mutual funds in the period 19451964. The Journal of Finance, 23(2), 389-416. https://doi.org/10.1111/j.1540$\underline{6261.1968 . t b 00815 . x}$

Jensen, M. C. (1969). Risk, the pricing of capital assets, and the evaluation of investment portfolios. The Journal of Business, 42(2), 167-247.

Lintner, J. (1965). The valuation of risk assets and the selection of risky investments in stock portfolios and capital budgets. The Review of Economics and Statistics, 47(1), 13-37. https://doi.org/10.2307/1924119

Malaquias, R., \& Maestri, C. (2017). Effects of Manager Characteristics on Portfolio Composition of Multimarket Funds. Revista Universo Contábil, 13(2), 89-108. https://doi.org/10.4270/RUC.2017210

Malkiel, B. G. (1995). Returns from investing in equity mutual funds 1971 to 1991. The Journal of Finance, 50(2), 549-572.

https://doi.org/10.2307/2329419

Matallín-Sáez, J. C., Soler-Domínguez, A., \& Tortosa-Ausina, E. (2018). Active management and mutual fund performance. Applied Economic Analysis, 26(78), 43-79.

Matos, P. R. F., Penna, C. M., \& Silva, A. B. G. (2015). Fundos Mútuos de Investimento em Ações no Brasil: Incentivos, Gestão e Convergência. Brazilian Business Review, 12(2), 115-149.

https://doi.org/10.15728/bbr.2015.12.2.6

Mossin, J. (1966). Equilibrium in a capital asset market. Econometrica: Journal of the Econometric Society, 768-783.

Riley, T. B. (2021). Portfolios of actively managed mutual funds. Financial Review, 1-26. https://doi.org/10.1111/fire.12257

Ross, S. A., Westerfield, R. W., \& Jaffe, J. F. (1995). Administração Financeira. São Paulo: Atlas. 
Sharpe, W. F. (1964). Capital asset prices: A theory of market equilibrium under conditions of risk. The Journal of Finance, 19(3), 425-442. https://doi.org/10.2307/2977928

Sharpe, W. F. (1966). Mutual fund performance. The Journal of Business, 39(1), 119-138.

Sharpe, W. F. (1991). The arithmetic of active management. Financial Analysts Journal, 47(1), 7-9.

Silva, S. E., Roma, C. M. da S., \& Iquiapaza, R. A. (2018). A Taxa de Administração Sinaliza o Desempenho dos Fundos de Investimento em Ações no Brasil?. Revista de Educação e Pesquisa Em Contabilidade (REPeC), 12(3), 286-302. https://doi.org/10.17524/repec.v12i3.1717

Silva, S. E., Roma, C. M. da S., \& Iquiapaza, R. A. (2020). Portfolio turnover and performance of equity investment funds in Brazil. Revista Contabilidade e Finanças, 31(83), 332-347. https://doi.org/10.1590/1808$\underline{057 \times 201909420}$

Stark, J. R. (2019). Decomposing mutual fund alpha into security selection and security weighting. Journal of Empirical Finance, 52, 76-91. https://doi.org/10.1016/j.jempfin.2019.03.001

Wermers, R. (2000). Mutual fund performance: An empirical decomposition into stock-picking talent, style, transactions costs, and expenses. The Journal of Finance, 55(4), 1655-1695. 